\title{
EFEITO DA TEMPERATURA E DE EMBALAGENS SOBRE A ANTRACNOSE EM FRUTOS DE MANGA cv. TOMMY ATKINS
}

\author{
Mayara Castro Assunção ${ }^{1 *}$, Ana Gabriele Gurgel Amaral ${ }^{2}$, Fernando Josias Alcântara Lins ${ }^{3}$ \\ ${ }^{1}$ Mestre em Proteção de Plantas, Centro de Ciências Agrárias, Universidade Federal de Alagoas, BR 104 Norte, Km 85, Rio Largo, AL. \\ CEP 57.000-100. \\ ${ }^{2}$ Discente de Pós-Graduação em Proteção de Plantas, Centro de Ciências Agrárias, Universidade Federal de Alagoas, BR 104 Norte, \\ Km 85, Rio Largo, AL. CEP 57.000-100. \\ ${ }^{3}$ Mestre em Agronomia (Produção Vegetal), Centro de Ciências Agrárias, Universidade Federal de Alagoas, BR 104 Norte, Km 85, Rio \\ Largo, AL. CEP 57.000-100. \\ *Autor para correspondência: Mayara Castro Assunção, mayara_castroa@hotmail.com
}

RESUMO: Este trabalho teve como objetivo avaliar a eficiência de diferentes temperaturas e embalagens para o controle da antracnose (Colletotrichum sp.) na cultura da manga. Os frutos selecionados encontravam-se em estádio de maturação comercial, possuindo uniformidade de cor, tamanho e ausência de injúrias mecânicas e fisiológicas, sendo submetidos à inoculação e em seguida à câmara úmida por 24 horas. Posteriormente, foram aplicados os tratamentos: $\mathrm{T} 1-10^{\circ} \mathrm{C}$ sem embalagem; $\mathrm{T} 2-10^{\circ} \mathrm{C}$ com embalagem individual; $\mathrm{T} 3-10^{\circ} \mathrm{C}$ com embalagem coletiva; $\mathrm{T} 4-24^{\circ} \mathrm{C}$ sem embalagem; $\mathrm{T} 5-24^{\circ} \mathrm{C}$ com embalagem individual; $\mathrm{T} 6-24^{\circ} \mathrm{C}$ com embalagem coletiva; $\mathrm{T7}$ - temperatura ambiente sem embalagem (testemunha); $\mathrm{T} 8$ - temperatura ambiente com embalagem individual; $\mathrm{T} 9$ - temperatura ambiente com embalagem coletiva. Utilizou-se delineamento inteiramente casualizado com cinco repetições, dispostos num esquema fatorial $3 \times 3$ (três temperaturas e três embalagens). Foram avaliadas incidência (I) e severidade (S) da doença aos 0, 4, 8 e 12 dias após os tratamentos (DAT). Os dados foram submetidos à análise de variância e as médias comparadas pelo teste de Scott-Knott $(P \leq 0,05)$. Os resultados obtidos demonstraram que todos os frutos analisados foram suscetíveis à antracnose, nos diferentes tratamentos, sendo 0 armazenamento refrigerado a $10^{\circ} \mathrm{C}$ com embalagem individual o que apresentou melhor resultado, podendo ser recomendado como um método alternativo no controle pós-colheita de antracnose em frutos de manga.

PALAVRAS-CHAVE: Mangifera indica, Colletotrichum sp., controle físico, pós-colheita

\section{EFFECT OF TEMPERATURE AND PACKAGING ON ANTHRACNOSE ON MANGO FRUITS cV. TOMMY ATKINS}

ABSTRACT: This work had as objective to evaluate the efficiency of different temperatures and packages for the control of anthracnose (Colletotrichum sp.) in mango culture. The fruits selected were commercially mature, with color uniformity, size and absence of mechanical and physiological injuries, being submitted to inoculation and then to the humid chamber for 24 hours. Subsequently, the treatments were applied: $\mathrm{T} 1-10^{\circ} \mathrm{C}$ without packaging; $\mathrm{T} 2$ $-10^{\circ} \mathrm{C}$ with individual packaging; $\mathrm{T} 3-10^{\circ} \mathrm{C}$ with collective packaging; $\mathrm{T} 4-24^{\circ} \mathrm{C}$ without packaging; $T 5-24^{\circ} \mathrm{C}$ with individual packaging; $\mathrm{T6}-24^{\circ} \mathrm{C}$ with collective packaging; $\mathrm{T7}$ - ambient temperature without packaging (control); $\mathrm{T} 8$ - ambient temperature with individual packaging; T9 - ambient temperature with collective packaging. A completely randomized design with five replicates, arranged in a factorial scheme $3 \times 3$ (three temperatures and three packages) was used. The incidence (I) and severity (S) of the disease were evaluated at $0,4,8$ and 12 days after treatments. The data were submitted to analysis of variance and the means compared by the Scott-Knott test ( $P \leq$ $0,05)$. The results showed that all fruits analyzed were susceptible to anthracnose in the different treatments, and the storage refrigerat at $10^{\circ} \mathrm{C}$ with individual packaging, which presented better results, and can be recommended as an alternative method for the post-harvest control of anthracnose in fruits of mango.

KEYWORDS: Mangifera indica, Colletotrichum sp., physical control, post harvest 


\section{INTRODUÇÃO}

A manga (Mangifera indica L.) é uma das mais importantes culturas tropicais produzidas no mundo e no Brasil, apresentando neste país, área cultivada de aproximadamente 64 mil hectares (Ibge, 2015). De acordo com os dados do lbge (2015), a produção nacional foi de 976.815 toneladas/ano, sendo a região Nordeste a maior produtora, com 45.238 t/ano e, o estado da Bahia com a máxima produção 21.130 t/ano.

Entretanto, parte do total produzido é perdido durante a fase de pós-colheita, de acordo com o Anuário Brasileiro de Fruticultura (2015) estima-se que o índice médio seja de $28 \%$, ocasionado por diversas causas, principalmente, dentre estas Colletotrichum sp., agente causador da antracnose; doença que afeta todas as partes da planta, sendo o prejuízo maior nos frutos, causando manchas escuras irregulares na casca, as quais podem coalescer e atingir grandes áreas. Geralmente, os frutos são infectados no campo pelo patógeno, e permanece latente até o estabelecimento de condições favoráveis ao seu desenvolvimento, comumente na maturação (Fischer et al., 2009a).

A antracnose ocorre, principalmente, nas regiões tropicais e subtropicais do mundo, podendo afetar os frutos do mamão (Carica papaya L.), goiaba (Psidium guajava L.), maracujá (Passiflora edulis Sims), banana (Musa spp.), abacate (Persea americana Mill.), manga e de uma ampla gama de hospedeiros, acarretando perdas de grande importância pois torna os frutos impróprios ao consumo e potencializa o desenvolvimento de saprófitas (Borges et al., 2016).

A antracnose é frequentemente causada por mais de uma espécie de Colletotrichum. 0 abacateiro e a goiabeira podem ser infectados por $C$. gloeosporioides (Penz.) Penz. \& Sacc. e C. acutatum J. H. Simmonds (Fischer et al., 2011; Tozze Júnior et al., 2015), 0 maracujazeiro pelas espécies $C$. gloeosporioides e $C$. boninense Moriwaki, Toy. Sato \& Tsukib (Tozze Júnior et al., 2015), a bananeira por C. musae (Santos et al., 2015), o mamoeiro por C. gloesporioides (Demartelaere et al., 2015), e para antracnose associada a mangueira são descritas até sete espécies deste gênero, $C$. gloeosporioides, C. acutatum, C. asianum Prihastuti, L. Cai \& K.D. Hyde, C. fructicola Prihastuti, L. Cai \& K.D. Hyde, C. tropicale Rojas, Rehner \& Samuels, C. karstii Y.L. Yang, Zuo Y. Liu, K.D. Hyde \& L. Cai e C. dianesei Lima, Nelson B. (Lima et al., 2013; Tozze Júnior et al., 2015).
Por se tratar de uma das doenças mais frequentes e responsáveis pelos maiores prejuízos em pós-colheita de manga, o manejo da antracnose tem se tornado indispensável, e o tratamento químico trata-se do método mais utilizado. No entanto, os fungicidas que são aplicados nas áreas de produção da cultura, devido 0 amplo espectro de ação e em função de seu uso contínuo, favorecem o desenvolvimento de patógenos resistentes e provocam risco de contaminação ao ambiente e aos frutos (Abd-AllA e Haggag, 2013; Lemos et al., 2013).

Em virtude da crescente exigência do mercado consumidor e da preocupação com a preservação do meio ambiente, devido a elevada quantidade de resíduo deixada pela aplicação de produtos químicos, métodos alternativos para o controle vêm sendo testados para que sejam, ao mesmo tempo, eficientes, minimizando os danos causados pelo patógeno, e menos nocivos à saúde humana e ao equilíbrio de ecossistemas, garantindo assim a segurança do produto (Thinh e Kunasakdakul, 2013).

Técnicas alternativas de controle tais como 0 uso de extratos de plantas (Borges et al., 2016), óleos essenciais (Abd-AllA e Haggag, 2013; Ramos et al., 2016), hidrotermia (Nascimento et al., 2014; Santos et al., 2011), radiação (Nascimento et al., 2014) e a utilização de revestimentos e embalagens (Borges et al., 2016; Santos et al., 2011) estão sendo aplicadas, uma vez que, não deixam resíduo ou apresentam menor poder residual, não colocam em risco a saúde dos consumidores e evitam a contaminação do meio ambiente. Sendo assim, o objetivo deste trabalho foi avaliar a eficiência de diferentes temperaturas e embalagens para o controle da antracnose ( $C$. gloeosporioides) na cultura da manga.

\section{MATERIAL E MÉTODOS}

Os frutos selecionados foram comprados em Centrais de Abastecimento, localizadas em Maceió, Alagoas, em estádio de maturação comercial, possuindo uniformidade de cor, tamanho e ausência de injúrias mecânicas e fisiológicas. Após a seleção, os mesmos foram levados para o laboratório de Fitopatologia da Embrapa Tabuleiros Costeiros (Unidade Rio Largo), onde passaram por assepsia, lavagem com água e sabão para desinfecção superficial e solução de hipoclorito a $0,05 \%$ por 20 minutos. 
$\mathrm{O}$ isolado de Colletotrichum sp. foi obtido do Laboratório de Fitopatologia do Centro de Ciências Agrárias, da Universidade Federal de Alagoas, oriundo de frutos de mangas e caracterizado morfoculturalmente. Discos de BDA (Batata-DextroseÁgar) contendo estruturas do fungo, preservados em água destilada, foram retirados e transferidos para 0 centro de placas de Petri contendo o mesmo tipo de meio, sendo cultivado, em condições ambientais ( \pm $26^{\circ} \mathrm{C}$ ), por sete dias. A caracterização morfológica foi realizada através do preparo de lâminas a partir de colônias do isolado, contendo conídios hialinos, unicelulares e em forma de bastonete, arredondados nas extremidades, o que identifica a presença do fungo Colletotrichum sp.

Posteriormente, os frutos de manga foram submetidos separadamente à inoculação com 0 fungo, através da colocação de discos de micélio do patógeno, retirados da extremidade de colônias jovens, sob ferimentos feitos com agulha esterilizada. Houve dois pontos de inoculação por fruto e em seguida, os mesmos foram colocados em câmara úmida (Alfenas et al., 2007).

Após 24 horas da inoculação, os frutos foram submetidos aos tratamentos $\mathrm{T} 1-10^{\circ} \mathrm{C}+$ embalagem individual; $\mathrm{T} 2-10^{\circ} \mathrm{C}+$ embalagem coletiva; T3 $10^{\circ} \mathrm{C}$ sem embalagem; $\mathrm{T} 4-24^{\circ} \mathrm{C}+$ embalagem individual; T5 - $24^{\circ} \mathrm{C}+$ embalagem coletiva; T6 $24^{\circ} \mathrm{C}$ sem embalagem; $\mathrm{T} 7$ - temperatura ambiente + embalagem individual; T8 - temperatura ambiente + embalagem coletiva; T9 - temperatura ambiente sem embalagem (testemunha). Os que possuíam embalagem individual eram envoltos por filme de PVC separadamente, e a embalagem coletiva consistia em envolver os cinco frutos, da repetição, em um único filme de PVC.

Para se avaliar o efeito dos tratamentos foram analisadas as seguintes variáveis: incidência, determinada pela média da quantidade de frutos com sintomas da doença, ou seja, foi avaliada a presença ou ausência de antracnose nos frutos de manga; e a severidade, medida com auxílio da escala diagramática de notas desenvolvida por Sales Júnior et al. (2004) para manga cv. Tommy Atkins.

A escala de notas adotada para avaliação da severidade variou de 1 a 5, com base na área lesionada, sendo as respectivas atribuições: 1. ausência de manchas de antracnose nos frutos; 2. menos de 5,0\% da área dos frutos afetados com antracnose; 3. de 5,0 - 10,0\% da área dos frutos afetados com antracnose; 4. de $10,1-30 \%$ da área dos frutos afetados com antracnose e 5. acima de 30,1\% da área dos frutos afetados com antracnose, sendo estas variáveis avaliadas aos 0, 4, 8 e 12 dias após a aplicação dos tratamentos (DAT).

Os tratamentos foram dispostos em um delineamento inteiramente casualizado num esquema fatorial $3 \times 3$, considerando-se três temperaturas e três embalagens, com cinco repetições. Os dados obtidos de incidência e severidade foram submetidos à análise de variância pelo teste $F$, constatando-se diferença significativa as médias foram comparadas pelo teste de Scott-Knott a 5\% de probabilidade com auxílio do programa estatístico SISVAR versão 5.3 (Ferreira, 2010).

\section{RESULTADOS E DISCUSSÃO}

Em todos os tratamentos avaliados houve incidência da doença após o período final avaliado. Na primeira avaliação, mesmo dia da aplicação dos tratamentos, não houve incidência da antracnose nos frutos. A partir da segunda avaliação, quatro dias após a aplicação dos métodos de controle, houve incidência da doença nos frutos e esta, manteve-se em aumento até o período final de avaliação (Figura 1). Entre os tratamentos, incluindo-se a testemunha, não houve diferença estatística para esta variável (Tabela 1). 
Figura 1. Incidência de antracnose (Colletotrichum sp.) em frutos de manga cv. Tommy Atkins durante 12 dias de avaliação.

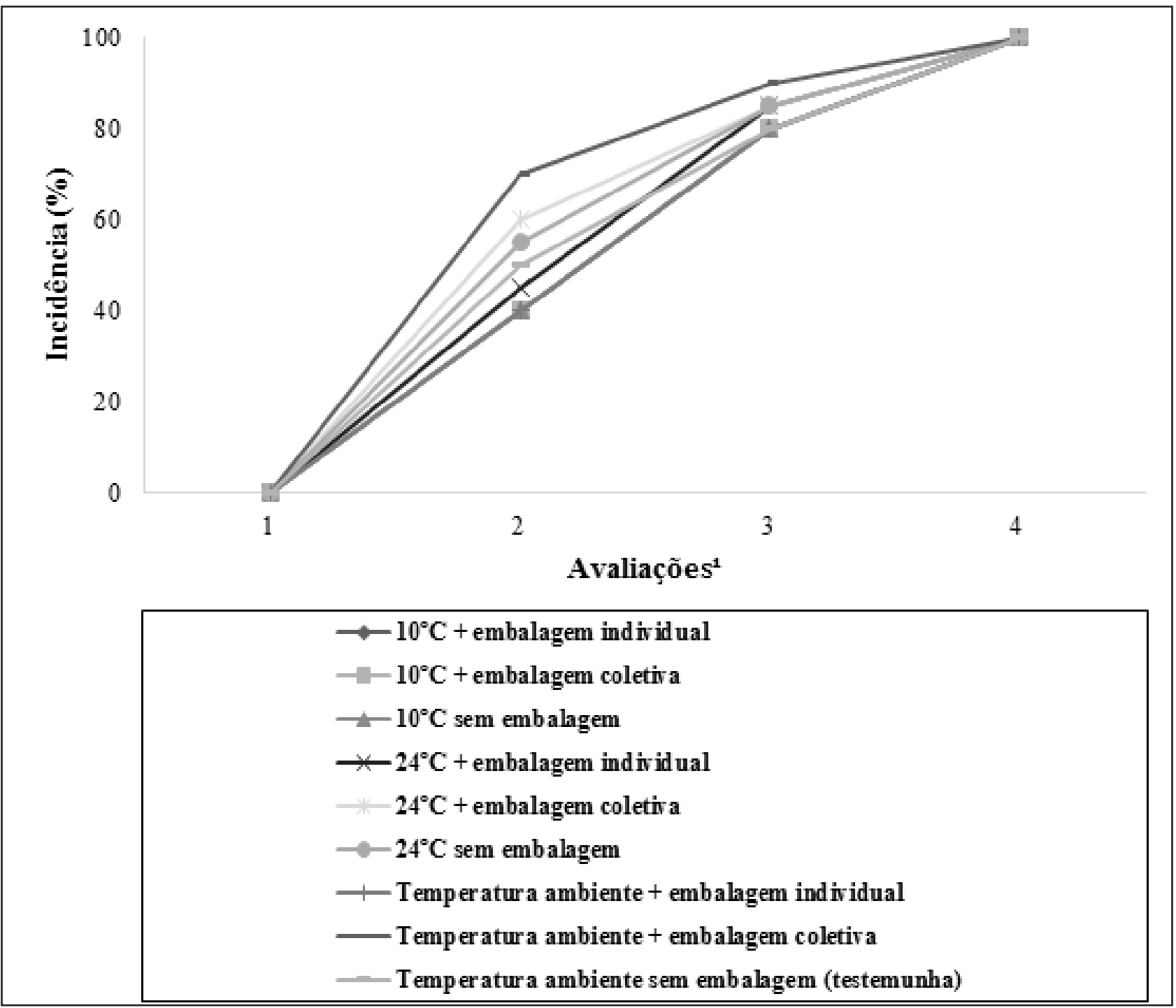

11: 0 DAT; 2: 4 DAT; 3: 8 DAT; 4: 12 DAT.

Tabela 1. Efeito de temperaturas e embalagens na incidência da antracnose em frutos de mangas cv. Tommy Atkins, durante 12 dias de avaliação.

\begin{tabular}{cc}
\hline Tratamento & Incidência (\%) \\
\hline $10^{\circ} \mathrm{C}+$ embalagem individual & $55,0 \mathrm{a}^{\mathrm{a}}$ \\
$10^{\circ} \mathrm{C}+$ embalagem coletiva & $55,0 \mathrm{a}$ \\
$10^{\circ} \mathrm{C}$ sem embalagem & $55,0 \mathrm{a}$ \\
$24^{\circ} \mathrm{C}+$ embalagem individual & $57,5 \mathrm{a}$ \\
$24^{\circ} \mathrm{C}+$ embalagem coletiva & $61,25 \mathrm{a}$ \\
$24^{\circ} \mathrm{C}$ sem embalagem & $60,0 \mathrm{a}$ \\
Temperatura ambiente + embalagem individual & $55,0 \mathrm{a}$ \\
Temperatura ambiente + embalagem coletiva & $65,0 \mathrm{a}$ \\
Temperatura ambiente sem embalagem (testemunha) & $57,5 \mathrm{a}$ \\
\hline $\mathrm{CV} \mathrm{( \% )} 8,95$ & \\
\hline
\end{tabular}

${ }^{a}$ Médias seguidas pelas mesmas letras não diferem entre si pelo teste de Scott-Knott $(P \leq 0,05)$. 
Avaliando a incidência da antracnose em frutos de manga na pós-colheita, Galli etal. (2012) constataram que ao final do período de armazenamento de 10 dias, todos os frutos apresentavam sintoma da doença. Fischer et al. (2009a) também obtiveram resultados semelhantes ao analisar a antracnose em pós-colheita de diferentes cultivares de manga, dentre estas Tommy Atkins, verificando a presença de C. gloeosporioides em $100 \%$ dos frutos estudados.

Populações do gênero Colletotrichum também foram relatadas em pesquisas realizadas nos frutos da goiaba, do mamão e do abacate, havendo maior incidência, para as três quantificações, maior incidência da antracnose. Nos frutos de mamão a frequência desta doença foi de $45 \%$, nos de abacate a incidência foi em média superior a $55 \%$, e no levantamento dos frutos de goiaba verificou-se que a doença de pós-colheita mais frequente foi a antracnose (Fischer et al., 2017; Galli et al., 2015; Rocha et al., 2015).

Em relação à severidade da antracnose, todos os tratamentos apresentaram índice a partir da segunda avaliação, quatro dias após a aplicação dos métodos de controle. Houve diferença significativa ( $P \leq$ $0,05)$ para as médias dentro dos tipos de embalagens e das temperaturas (Tabela 2).

Tabela 2. Valores médios de severidade de mangas cv. Tommy Atkins, após tratamento sob diferentes temperaturas e embalagens, durante 12 dias de avaliação.

\begin{tabular}{cccc}
\hline \multirow{2}{*}{ Temperatura } & \multicolumn{3}{c}{ Tipo da Embalagem } \\
\cline { 2 - 4 } & Embalagem individual & Embalagem coletiva & Sem embalagem \\
\hline $10^{\circ} \mathrm{C}$ & $1,50 \mathrm{aA}$ & $1,50 \mathrm{aA}$ & $1,50 \mathrm{aA}^{a}$ \\
$24^{\circ} \mathrm{C}$ & $1,75 \mathrm{aA}$ & $3,00 \mathrm{bB}$ & $2,50 \mathrm{bA}$ \\
Temperatura Ambiente & $1,50 \mathrm{aA}$ & $3,25 \mathrm{bB}$ & $2,25 \mathrm{aA}$ \\
\hline $\mathrm{CV}(\%)$ & 31,75 & &
\end{tabular}

aMédias seguidas pelas mesmas letras, minúsculas nas linhas, e maiúsculas nas colunas, não diferem entre si pelo teste de Scott-Knott $(P \leq 0,05)$.

Quando consideradas as temperaturas, constatou-se diferença significativa em $24^{\circ} \mathrm{C}$ e na temperatura ambiente. Para $24^{\circ} \mathrm{C}$, a embalagem individual apresentou menor média de severidade, 1,75, diferindo da sem embalagem e da embalagem coletiva, com médias 2,50 e 3,0, respectivamente. Natemperatura ambiente, a embalagem coletiva apresentou maior média, 3,25, divergindo estatisticamente das demais, com médias de 2,25 para frutos sem embalagem e 1,50 para embalagem individual.

Em trabalho desenvolvido por (Miguel et al., 2011), avaliando a severidade de antracnose em frutos de manga cv. Palmer, dentre as temperaturas, $10^{\circ} \mathrm{C}$ foi a que demonstrou menores médias para todas as embalagens analisadas. Galli et al. (2013) observaram que 0 armazenamento a $12^{\circ} \mathrm{C}$ para mangas cv. Espada foi o mais eficiente para o controle do desenvolvimento de C. gloesporioides, diminuindo a severidade da doença, e consequentemente, controlando-a.

Para os frutos maracujá-amarelo e amora preta, o armazenamento refrigerado trata-se também de uma alternativa eficaz para o controle da antracnose, mantendo a qualidade de frutos, com temperaturas ideias de $8^{\circ} \mathrm{C}$ e de $2^{\circ} \mathrm{C}$ a $5^{\circ} \mathrm{C}$, respectivamente, apresentando menores índices de severidade (Palharini et al., 2015; Weber et al.; 2012). Os resultados obtidos nesses estudos, ratificam o resultado encontrado na presente pesquisa, visto que as baixas temperaturas se despontam mais eficientes para controle de podridões de pós-colheita.

A refrigeração diminui a taxa respiratória, interrompe o metabolismo, possui efeito microbiostático e retarda 0 amadurecimento, consequentemente, suprime o desenvolvimento de patógenos, entretanto, o período de exposição a refrigeração deve ser considerado, pois se muito extenso, pode facilitar a ocorrência de outras doenças (Miguel et al. 2013), além de causar injúrias como enrugamento e escurecimento da casca, devido à perda de água, e o murchamento da polpa, o que afeta o aspecto visual e reduz o valor de comércio.

Para os tipos de embalagem apenas a coletiva apresentou significância, com menor severidade na temperatura de $10^{\circ} \mathrm{C}$, média de 1,50 , diferindo de $24^{\circ} \mathrm{C}$ e da temperatura ambiente, com médias 3,0 e 3,25 , respectivamente. A embalagem que obteve 
melhor resultado, independente da temperatura, fo a individual, com médias 1,$50 ; 1,75 ; 1,50$ para $10^{\circ} \mathrm{C}$, $24^{\circ} \mathrm{C}$ e temperatura ambiente, respectivamente. Entretanto, este tipo de embalagem não diferenciou significativamente dos frutos que não possuíam o filme de PVC, para $10^{\circ} \mathrm{C}$ e temperatura ambiente, e para embalagem coletiva à $10^{\circ} \mathrm{C}$.

Resultados semelhantes foram obtidos por Jerônimo et al. (2007) verificando a conservação póscolheita de mangas cv. Tommy Atkins em condições de temperatura ambiente e atmosfera modificada com a utilização de diferentes tipos de embalagens, sendo os tratamentos de atmosfera modificada com os frutos individualmente recobertos com o filme de PVC também os que apresentaram melhores resultados.

Rodrigues et al. (2008) ao analisar a vida útil de mangas armazenadas em embalagem com atmosfera modificada e em temperatura ambiente, também constatou que as amostras acondicionadas nas condições de temperatura ambiente apresentam maiores contagens de fitopatógenos, ou seja, maior severidade de doenças, do que as acondicionadas em embalagens.

Em trabalho realizado por Lima et al. (2007) e Santos et al. (2011), o uso de embalagens plásticas de PVC, associado ao armazenamento refrigerado, também foi eficiente na conservação pós-colheita de cultivares de manga, dentre estas 'Tommy Atkins'. Além de diminuir o índice de infecção da antracnose, frutos embalados e refrigerados a $10^{\circ} \mathrm{C}$ mantem por mais tempo as características ótimas para o consumo desta cultura (Lima et al., 2007).

A utilização de filmes plásticos em estudo realizado por Prill et al. (2012) indicou resultado positivo no aparecimento de lesões nas cascas dos frutos de banana 'Prata-Anã', pois retardou o processo de maturação e proporcionou a proteção dos frutos contra patógenos de pós-colheita, permitindo a ampliação do período de comercialização.

Em pesquisa executada por Morais et al. (2010) avaliando a influência da atmosfera modificada sob a vida útil pós-colheita do mamão cv. Formosa, os frutos embalados apresentaram menores danos na casca, mesmo havendo a incidência de podridões, ressaltando os resultados encontrados no presente estudo, porém, Arruda et al. (2011) obtiveram em seu trabalho que o filme PVC individualizado não foi suficientemente eficiente para o controle de patógenos pós-colheita, dentre estes Colletotrichum spp., no maracujá-amarelo.

0 uso da refrigeração com embalagens durante 0 armazenamento dos frutos, prolonga 0 período de conservação, pois a embalagem cria um tipo de barreira artificial e evita assim os danos causados, principalmente, por podridões. Sendo assim, é extremamente importante o emprego de embalagens adequadas no tratamento dos frutos, pois permite uma melhor conservação e manutenção da qualidade na pós-colheita, facilitando sua exportação e comercialização (Paiva et al., 2012).

Os resultados obtidos demonstram que a refrigeração na temperatura de $10^{\circ} \mathrm{C}$ com o uso de embalagem individual pode ser utilizada como um método alternativo no controle de Colletotrichum sp. em frutos de manga, visando redução de perdas durante a pós-colheita.

\section{REFERÊNCIAS BIBLIOGRÁFICAS}

Abd-AllA, M. A.; Haggag, W. M. Use of Some Plant Essential Oils as Post-harvest Botanical Fungicides in the Management of Anthracnose Disease of Mango Fruits (Mangi Feraindica L.) Caused by Colletotrichum Gloeosporioides (Penz). International Journal of Agriculture and Forestry, 2013, 3, 1, 1-6.

Alfenas, A. C.; Mafia, R. G. Métodos em Fitopatologia. Edição 22; Universidade Federal de Viçosa: Viçosa, MG, 2007; 382p.

Anuário Brasileiro de Fruticultura, Reetz, E. R.; Kist, B. B.; Santos, C. E.; Carvalho, C.; Drum, M., Santa Cruz do Sul: Editora Gazeta Santa Cruz, 2015. 104p.

Arruda, M. C.; Fischer, I. H.; Jeronimo, E. M.; Zanett, M. M.; Silva, B. L. Efeito de produtos químicos e temperaturas de armazenamento na pós-colheita de maracujá-amarelo. Ciências Agrárias, 2011, 32, 1, 201 207.

Borges, I. V.; Cavalcanti, L. S.; Figueirêdo Neto, A.; Almeida, J. R. G. S.; Rolim, L. A.; Lima, M. A. G. Aplicação de revestimento com extratos de Jurema Preta no controle de antracnose em frutos de manga. Revista Iberoamericana de Tecnología Postcosecha. 2016, 17, 2, 205-216. 
Demartelaere, A. C. F.; Guimarães, G. H. C.; Silva, J. A.; Luna, R. G.; Nascimento, L. C. Extratos vegetais no controle da antracnose e na conservação da qualidade em frutos de mamoeiro. Revista Brasileira de Plantas Medicinais, 2015, 17, 4, 1041-1048.

Ferreira, D. F. SISVAR. Universidade Federal de Lavras-MG. Versão 5.3 (Build 77), 2010.

Fischer, I. H.; Almeida, A. M.; Arruda, M. C.; Bertani, R. M. A.; Garcia, M. J. M.; Amorim, L. Danos em pós-colheita de goiabas na Região do Centro-Oeste Paulista. Bragantia, 2011, 70, 3, 570-576.

Fischer, I. H.; Arruda, M. C.; Almeida, A. M.; Montes, S. M. N. M..; Jerônimo, E. M.; Simionato, E. M. R. S.; Bertani, R. M. A. Doenças e características físicas e químicas pós-colheita em manga. Ciências Agrárias, 2009a, 30, 1, 107-116.

Fischer, I. H.; Moraes, M. F.; Palharini, M. C. A.; Cruz, J. C. S.; Firmino, A. C. Ocorrência de antracnose em abacate, agressividadee sensibilidade de Colletotrichum gloeosporioides a fungicidas. Agropecuária Científica no Semiárido Centro de Saúde e Tecnologia Rural, 2017, 13, 2, 130-137.

Galli, J. A.; Fischer, I. H.; Palharini, M. C. A. Doenças pré e pós-colheita em variedades de manga cultivadas em sistema orgânico. Revista Brasileira de Fruticultura, 2012, 34, 3, 734-743.

Galli, J. A.; Fischer, I. H.; Palharini, M. C. A.; Michelotto, M. D. Quantificação de doenças pós-colheita em acessos de goiabeira cultivados em sistema orgânico. Pesquisa Agropecuária Tropical, 2015, 45, 2, 225-230.

Galli, J. A.; Soares, M. B. B.; Martins, A. L. M.; Galli, J. C. Storage of 'Espada' mango fruits in modified atmosphere and cooling: effects on conservation. Fruits, 2013, 68, 291-302.

IBGE, Produção Agrícola Municipal. (http:// biblioteca.ibge.gov.br/índex.Php/biblioteca -catalogo?view=detalhes\&id=766) 07 de junho de 2017.

Jerônimo, E. M.; Brunini, M. A.; Arruda, M. C.; Cruz, J. C. S.; Fischer, I. H.; Gava, G. J. C. Conservação póscolheita de mangas 'Tommy Atkins' armazenadas sob atmosfera modificada. Ciências Agrárias, 2007, 28, 3, 417-426.

Lemos, L. M. C.; Coutinho, P. H.; Salomão, L. C. C.; Siqueira, D. L.; Cecon, P. R. Controle da antracnose na pós-colheita de manga 'ubá' com o uso de produtos alternativos. Revista Brasileira de Fruticultura, 2013, $35,4,962-970$.

Lima, L. C.; Dias, M. S. C.; Castro, M. V.; Ribeiro Júnior, P. M.; Silva, E. B. Controle da antracnose e qualidade de mangas (Mangifera indica L.) CV. Haden, após tratamento hidrotérmico e armazenamento refrigerado em atmosfera modificada. Ciência e Agrotecnologia, 2007, 31, 2, 298-304.

Lima, N. B.; Batista, M. V. A.; Morais Júnior, M. A.; Barbosa, M. A. G.; Michereff, S. J.; Hyde, K. D.; Câmara, M. P. S. Five Colletotrichum species are responsible for mango anthracnose in northeastern Brasil. Fungal Diversity, 2013, 61, 1, 75-88.

Miguel, A. C. A.; Durigan, J. F.; Barbosa, J. C.; Morgado, C. M. A. Qualidade de mangas cv. Palmer após armazenamento sob baixas temperaturas. Revista Brasileira de Fruticultura, 2013, 35, 2, 398- 408.

Miguel, A. C. A.; Durigan, J. F.; Morgado, C. M. A.; Gomes, R. F. O. Injúria pelo frio na qualidade póscolheita de mangas cv. Palmer. Revista Brasileira de Fruticultura, 2011, 255-260.

Morais, F. A.; Araújo, F. M. M. C.; Machado, A. V.; Ricarte, F. D. N.; Sales Junior, R. Influência da atmosfera modificada sob a vida útil pós-colheita do mamão 'formosa'. Revista Verde, 2010, 5, 4, 01 - 09.

Nascimento, F. V.; Santos, M. C.; ValdebenitoSanhueza, R. M.; Bartnicki, V. A. Hidrotermia e radiação UV-C no controle de patógenos de manga e melão. Summa Phytopathologica, 2014, 40, 4, 313-317.

Paiva, A.; Lee, A.; Kowalski, M.; Silva, O. Chilling e Freesing - Injúrias causadas por baixas temperaturas em frutas tropicais. Universidade de São Paulo - USP, 2012, 1 Ed., p.14.

Palharini, M. C. A.; Fischer, I. H.; Vegian, M. R. C.; Fileti, M. S.; Montes, S. M. N. M. Efeito da temperatura 
de armazenamento na conservação pós-colheita de amora-preta. Pesquisa Agropecuária Tropical, 2015, 45, 4, 413-419.

Prill, M. A. S.; Neves, L. T. B. C.; Campos, A. J.; Silva, S.; Chagas, E. A.; Araújo, W. F. Aplicações de tecnologias pós-colheita para bananas 'Prata-Anã' produzidas em Roraima. Revista Brasileira de Engenharia Agrícola e Ambiental, 2012, 16, 11, 1237-1242.

Ramos, K.; Andreani Júnior, R.; Kozusny-Andreani, D. I. Óleos essenciais e vegetais no controle in vitro de Colletotrichum gloeosporioides. Revista Brasileira de Plantas Medicinais, 2016, 18, 2 (Supl. I), 605-612.

Rocha, R. H. C.; Cezar, M. A.; Satiro, D. D. S.; Silva, H. S.; Sousa, F. A. Características físico-químicas e incidência de patógenos fúngicos em mamão formosa comercializado no sertão paraibano. Revista Brasileira de Produtos Agroindustriais, 2015, 17, 2, 199-205.

Rodrigues, L. K.; Pereira, L. M.; Ferrari, C. C.; Sarantópoulos, C. I. G. L.; Hubinger, M. D. Vida útil de fatias de manga armazenadas em embalagem com atmosfera modificada passiva. Ciência e Tecnologia de Alimentos, 2008, 28 (Supl.), 271-278.

Sales Júnior, R.; Costa, F. M.; Marinho, R. E. M.; Nunes, G. H. S.; Amaro Filho, J.; Miranda, V. S. Utilização de azoxistrobina no controle da antracnose da mangueira. Fitopatologia Brasileira, 2004, 29, 2, 193-196.
Thinh, D. C.; Kunasakdakul, K. Inhibition of Colletotrichum gloeosporioides and Control of Postharvest Anthracnose Disease on Mango Fruit Using Propionic Acid Combined with Bee-Carnauba Wax Emulsion. Journal of Agricultural Science, 2013, $5,12,110-116$.

Tozze Júnior, H. J.; Firmino, A. C.; Fischer, I. H.; Furtado, E. L.; Massola Júnior, N. S. Caracterização de isolados de Colletotrichum spp. associados às frutíferas no Estado de São Paulo. Summa Phytopathologica, 2015, 41, 4, 270-280.

Santos, A. E. O.; Gravina, G. A.; Berbert, P. A.; Batista, P. F.; Santos, O. O. Efeito do tratamento hidrotérmico e diferentes revestimentos na conservação pós-colheita de mangas 'Tommy Atkins'. Revista Brasileira de Ciências Agrárias, 2011, 6, 1, 140-146.

Santos, P. C. M.; Lima, W. G.; Bezerra, C. S.; Michereff, S. J.; Câmara, M. P. S. Diversidade genotípica e patogênica de Colletotrichum musae no estado de Pernambuco. Revista Brasileira de Fruticultura, 2015, 37, 2, 355-366.

Weber, D.; Jones, E.; Beskow, G. T.; Barbosa, M. M.; Saavedra del Aguila, J.; Fachinello, J. C. Ácido salicílico e refrigeração na conservação de maracujás. Revista Iberoamericana de Tecnologia Postcosecha, 2012, 13, 2, 123-129. 